\title{
SOBREVIVÊNCIA DE CONÍDIOS DE Mycosphaerella fijiensis EM DIFERENTES MATERIAIS
}

\author{
ROGÉRIO E. HANADA ${ }^{1}$, LUADIR GASPAROTTO ${ }^{2} \&$ J. CLÉRIO R. PEREIRA ${ }^{2}$
}

\author{
${ }^{1}$ Coordenação de Pesquisas de Produtos Florestais - Instituto Nacional de Pesquisas da Amazônia, \\ Cx. Postal 478, CEP 69011-970, Manaus, AM, Fax: (92) 643-3096, e-mail: rhanada@inpa.gov.br; \\ ${ }^{2}$ Embrapa Amazônia Ocidental, Cx. Postal 319, CEP 69011-970, Manaus, AM
}

(Aceito para publicação em 02/04/2002)

Autor para correspondência: Rogério Eiji Hanada

HANADA, R.E., GASPAROTTO, L. \& PEREIRA, J.C.R. Sobrevivência de conídios de Mycosphaerella fijiensis em diferentes materiais. Fitopatologia Brasileira 27:408-411. 2002.

\begin{abstract}
RESUMO
Determinou-se o período de sobrevivência de conídios de Mycosphaerella fijiensis sobre diversos materiais como: madeira, plástico, tecido de algodão, papelão, pneu, ferro (carcaça de automóvel), folhas e frutos de bananeira (Musa sp.), materiais possíveis de transportar e disseminar o patógeno a longas distâncias. Concomitantemente, avaliou-se a sobrevivência de $M$. fijiensis associada a folhas de bananeira com mais de $50 \%$ da área foliar lesionada. Os materiais foram infestados com conídios de M. fijiensis, em locais predeterminados, produzidos em meio de BDA. Os materiais, as folhas e os frutos infestados e as folhas com sintomas da doença, foram mantidos em sala com condicionador de ar $(17,8$ $20,1{ }^{\circ} \mathrm{C}$ e 40 - 50\% U.R.), em sala com temperatura ambiente (23,6 $29,8{ }^{\circ} \mathrm{C}$ e 55 - $75 \%$ U.R.) e também em um galpão em condições de

campo $\left(22,2-30,9{ }^{\circ} \mathrm{C}\right.$ e 60 - 92\% U.R.). As avaliações foram feitas imediatamente após a infestação e com um, três, cinco, sete, dez, 13, 18, 23, 30 e 60 dias, removendo-se os conídios e semeando-os em placas de Petri contendo ágar-água, mantidas em incubadora a $25{ }^{\circ} \mathrm{C}$ $\pm 2{ }^{\circ} \mathrm{C}$, no escuro. Após $24 \mathrm{~h}$, avaliou-se, sob microscópio ótico, a germinação dos conídios. O comportamento da sobrevivência dos conídios nos diferentes materiais e associados nas folhas doentes, foi semelhante nos três ambientes testados. Os conídios de $M$. fijiensis permaneceram viáveis até a última avaliação (60 dias) em folhas de bananeira e tecido de algodão; até 30 dias em papelão, madeira, plástico e pneu; até 18 dias em frutos e até dez dias em ferro.

Palavras-chave adicionais: sobrevivência, Sigatoka negra, bananeira.

\section{ABSTRACT \\ Conidium survival of Mycosphaerella fijiensis on different materials}

An experiment was conducted with the objective to determine the survival period of Mycosphaerella fijiensis on common long distance dispersal materials: iron, wood, plastic, cotton textiles, fruits and leaves of banana (Musa sp.), rubber (tires) and cardboard packing. Conidia of M. fijiensis produced on PDA were atomized onto materials. The infested materials were placed in rooms with and without air conditioning systems and in a shed. Conidium survival was evaluated zero, one, three, five, seven, ten, 13, 18, 23,

30 and 60 days after infestation by collecting conidia, plating them and incubating at $25{ }^{\circ} \mathrm{C}$. Conidium germination was quantified by microscope $24 \mathrm{~h}$ after incubation. The results showed that the conidia remained viable on leaves and cotton textiles until the last evaluation. The viability of $M$. fijiensis on cardboard, wood, plastic and tires surfaces was detected up to 30 days after infestation. On fruits and iron, conidia remained viable until 18 days and ten days after infestation, respectively.
\end{abstract}

Em 1998, a Sigatoka negra da bananeira (Musa sp.) causada por Mycosphaerella fijiensis Moleret foi registrada no Brasil, nos municípios de Tabatinga e Benjamim Constant, estado do Amazonas (Pereira et al., 1998). Atualmente, o patógeno encontra-se disseminado por todo o estado do Amazonas, Rondônia, Acre, parte de Mato Grosso (região de Cárceres) e mais recentemente foi constatado nos municípios de Tartarugalzinho - Amapá, Caruebe - Roraima e Almerim Pará (Gasparotto et al., 2001). Nos demais estados produtores de banana do país, há uma preocupação generalizada quanto à introdução desse patógeno. Diante desse fato, as autoridades de vários estados livres da doença estão emitindo, ou já emitiram, portarias impedindo a entrada de mudas e frutas de bananeira oriundas dos estados atingidos pela doença.
A disseminação de propágulos férteis do patógeno é o processo responsável pelo incremento da doença (Amorim, 1995). Mycosphaerella fijiensis produz quantidade significativa de propágulos que são dispersos pelo vento. Os ascosporos desenvolvidos em grande quantidade no interior dos peritécios têm sido considerados o principal propágulo de disseminação de M. fijiensis em bananais (Gonzáles, 1999; Stover, 1980). Entretanto, conídios também podem ser considerados estruturas de disseminação do patógeno (Jacome \& Schuh, 1993a, 1993b; Gonzáles, 1999).

Apesar de o vento ser considerado um dos principais meios de disseminação dos esporos do fungo, as mudas doentes e as folhas infetadas, que são colocadas entre os cachos para evitar o ferimento dos frutos durante o transporte, 
também podem se constituir um meio eficiente e rápido para a disseminação do patógeno a longas distâncias. Além disso, os esporos quando aderidos nas embalagens, principalmente caixas, nos próprios frutos, nas roupas dos operários e nos veículos, dependendo do seu período de sobrevivência, podem ser transportados para áreas livres da doença. Diante desse fato, objetivou-se neste trabalho avaliar a sobrevivência de conídios de $M$. fijiensis aderidos a diferentes materiais e associados a lesões em folhas doentes destacadas.

O trabalho foi conduzido no Laboratório de Fitopatologia da Embrapa Amazônia Ocidental e no Laboratório de Patologia de Madeira, da Coordenação de Pesquisas de Produtos Florestais, Instituto Nacional de Pesquisas da Amazônia (INPA), em Manaus, AM.

A sobrevivência dos conídios do patógeno foi avaliada em amostras de folhas de bananeira, cascas de bananas verdes até ao amadurecimento, pedaços de madeira, papelão, plástico, tecido de algodão, ferro (carcaça de veículos) e pneu, com dimensões aproximadas de $10 \times 15 \mathrm{~cm}$.

Os esporos foram produzidos a partir do isolado LPM 472, em meio de cultura BDA (Hanada et al., 2002). Em seguida, foram coletados em água destilada, centrifugados por $2 \mathrm{~min}$ a $3.000 \mathrm{rpm}$, descartando-se o sobrenadante, e do precipitado preparou-se uma suspensão de 9,6 x $10^{6}$ conídios/ $\mathrm{ml}$. Foram distribuídos cerca de $0,04 \mathrm{ml}$ da suspensão sobre os materiais, com auxílio de um pincel, em círculos de aproximadamente $0,5 \mathrm{~cm}$ de diâmetro e em locais prédeterminados.

Os materiais contendo os conídios de M. fijiensis foram colocados em bandejas e distribuídos em sala com condicionador de ar (17,8 - 20,1 ${ }^{\circ} \mathrm{C}$ e 40 - 50\% U.R.), em sala com temperatura ambiente $\left(23,6-29,8^{\circ} \mathrm{C}\right.$ e $55-75 \%$ U.R.) e, também, em um galpão em condições de campo (22,2 $30,9^{\circ} \mathrm{C}$ e 60 - $92 \%$ U.R.). Em cada ambiente foram colocados quatro repetições de cada material contendo conídios do patógeno.

Avaliou-se a germinação dos conídios aos zero, um, dois, três, cinco, sete, 10, 13, 18, 23, 30 e 60 dias, após a sua deposição em cada material. Os conídios foram transferidos, por meio de raspagem com um estilete flambado, para placas de Petri contendo ágar-água $2 \%$, mantidas em incubadora a $25{ }^{\circ} \mathrm{C} \pm 2{ }^{\circ} \mathrm{C}$, no escuro $24 \mathrm{~h}$. Após esse período, foram observadas presença ou ausência de tubo germinativo de 100 conídios de cada parcela, perfazendo um total de 400 conídios por tratamento, sob microscópio óptico.

Também se avaliou a sobrevivência de $M$. fijiensis associada às lesões em folhas doentes destacadas. Para tanto, foram coletadas na área experimental da Embrapa Amazônia Ocidental, folhas doentes da cultivar Prata Anã, apresentando mais de $50 \%$ da área foliar lesionada. Quinze amostras de 20 $\mathrm{cm}$ x $30 \mathrm{~cm}$ foram distribuídas em locais semelhantes aos dos materiais em teste e a viabilidade do patógeno nas amostras foi avaliada nos mesmos intervalos de tempo. Sob microscópio estereoscópico, conídios foram transferidos com estilete de ponta fina para placas de Petri contendo meio de BDA, que foram mantidas à temperatura de $25^{\circ} \mathrm{C} \pm 2{ }^{\circ} \mathrm{C}$.
Diariamente, observou-se a formação de colônias no meio de cultura.

A sobrevivência dos conídios nos diferentes materiais foi semelhante nas três condições de ambientes testados (sala com condicionador de ar, sala com temperatura ambiente e galpão em condições de campo). Os conídios permaneceram viáveis até à última avaliação (60 dias) em folhas de bananeira e tecido de algodão; até 30 dias em papelão, madeira, plástico e pneu; até 18 dias nos frutos, devido ao seu apodrecimento, e até dez dias no ferro, provavelmente devido à oxidação do material (Tabela 1). Pelo fato de não haver diferenças de germinação de conídios de $M$. fijiensis independentemente dos efeitos ambientais, utilizaram-se os valores médios, nos três ambientes testados, para determinar a curva de sobrevivência dos conídios (Figura 1).

Portanto, os conídios aderidos à superfície de caixas de papelão, plástico ou madeira, tecidos das roupas dos operários; carrocerias, cabine e pneus de veículos que transitam em bananais afetados por Sigatoka negra, podem ser um agente de disseminação a longa distância, independentemente de transportar o fruto. Estes resultados sobre a longevidade dos conídios de $M$. fijiensis, na ausência do hospedeiro, são informações valiosas do ponto de vista epidemiológico, com implicações diretas na disseminação do patógeno.

Todavia, os conídios associados às lesões das folhas coletadas no campo permaneceram viáveis até à última avaliação (60 dias) nos três ambientes testados. Krans et al. (1982) citam que conídios de M. musicola e M. fijiensis, em condições naturais, podem sobreviver de três a quatro semanas associados às folhas doentes e ascosporos oito semanas no interior de peritécios. Os mesmos períodos de sobrevivência dos ascosporos associados em folhas de bananeiras caídas no chão foram relatados por Stover (1980). Esses dados reforçam os resultados obtidos neste trabalho, uma vez que, os conídios de $M$. fijiensis sobreviveram em folhas destacadas até 60 dias.

Alguns fungos produzem esporos que apresentam certas características que mantêm a sua viabilidade por um determinado período. Segundo Sussman \& Halvarson (1966), esses esporos possuem alta pressão osmótica, o que os permite absorver água do ar e manter sua viabilidade por um longo período. É provável que conídios de M. fijiensis sejam um desses esporos cujas características permitem uma maior longevidade, explicando os dados registrados nesse trabalho.

Pode existir alguma desidratação dos conídios sob as condições de ar-condicionado o que mostra que esporos de M. fijiensis podem sobreviver mesmo quando expostos a algum grau de desidratação. Esse comportamento de esporos foi, também, constatado para alguns fungos como Drechslera spp. (Dhingra \& Sinclair, 1995), Erysiphe graminis DC. f. sp. hordei Em. Marchal., (Moseman \& Powers, 1957) e ferrugem em folhas de trigo (Triticum aestivum L.) (Young \& Wadsworth, 1953).

A presença e a sobrevivência de conídios de M. fijiensis em folhas doentes reforçam a recomendação de que folhas de bananeira não devem ser utilizadas na proteção dos frutos 
R.E. Hanada et al.

TABELA 1 - Percentagem de germinação de conídios de Mycosphaerella fijiensis

\begin{tabular}{|c|c|c|c|c|c|c|c|c|}
\hline \multirow{2}{*}{ Material } & \multirow{2}{*}{ Condição } & \multicolumn{7}{|c|}{ Avaliação (dias) } \\
\hline & & 0 & 1 & 5 & 10 & 18 & 30 & 60 \\
\hline \multirow{3}{*}{$\begin{array}{l}\text { Folha de } \\
\text { bananeira }\end{array}$} & Com Ar & $87 *$ & 78,5 & 58,2 & 45,2 & 30,5 & 13,7 & 5,7 \\
\hline & Sem Ar & 87 & 76,0 & 59,5 & 48,2 & 30,5 & 13,5 & 3,7 \\
\hline & Campo & 87 & 77,5 & 58,2 & 45,2 & 30,7 & 18,0 & 8,0 \\
\hline \multirow{3}{*}{ Tecido } & Com Ar & 87 & 79,5 & 67,7 & 60,5 & 43,5 & 26,5 & 5,2 \\
\hline & Sem Ar & 87 & 79,2 & 61,2 & 40,0 & 33,7 & 12,5 & 1,0 \\
\hline & Campo & 87 & 77,5 & 59,2 & 45,0 & 33,0 & 17,5 & 1,7 \\
\hline \multirow{3}{*}{ Papelão } & Com Ar & 87 & 75,0 & 36,0 & 24,5 & 13,5 & 2,7 & 0,0 \\
\hline & Sem Ar & 87 & 76,5 & 36,8 & 31,5 & 16,2 & 2,2 & 0,0 \\
\hline & Campo & 87 & 76,5 & 57,0 & 31,2 & 15,0 & 2,5 & 0,0 \\
\hline \multirow{3}{*}{ Pneu } & Com Ar & 87 & 75,5 & 55,5 & 38,0 & 17,5 & 0,5 & $\overline{0,0}$ \\
\hline & Sem Ar & 87 & 74,5 & 55,2 & 37,7 & 18,2 & 0,7 & 0,0 \\
\hline & Campo & 87 & 74,2 & 55,0 & 38,2 & 14,7 & 0,7 & 0,0 \\
\hline \multirow{3}{*}{ Plástico } & Com Ar & 87 & 76,2 & 42,5 & 23,2 & 10,7 & 0,5 & 0,0 \\
\hline & Sem Ar & 87 & 77,7 & 43,5 & 27,0 & 11,7 & 0,5 & 0,0 \\
\hline & Campo & 87 & 75,0 & 45,5 & 28,2 & 11.0 & 1,0 & 0,0 \\
\hline \multirow{3}{*}{ Madeira } & Com Ar & 87 & 74,2 & 53,0 & 39,7 & 28,0 & 5,0 & 0,0 \\
\hline & Sem Ar & 87 & 74,5 & 56,5 & 40,0 & 29,5 & 5,5 & 0,0 \\
\hline & Campo & 87 & 77,5 & 51,2 & 38,7 & 29,0 & 3,2 & 0,0 \\
\hline \multirow{3}{*}{ Fruto } & Com Ar & 87 & 79,0 & 53,0 & 27,5 & 10,7 & 0,0 & 0,0 \\
\hline & Sem Ar & 87 & 78,2 & 61,0 & 26,5 & 10,2 & 0,0 & 0,0 \\
\hline & Campo & 87 & 80,2 & 59,2 & 24,7 & 10,5 & 0,0 & 0,0 \\
\hline \multirow{3}{*}{ Ferro } & Com Ar & 87 & 71,5 & 19,2 & 0,5 & 0,0 & 0,0 & 0,0 \\
\hline & Sem Ar & 87 & 73,5 & 20,2 & 0,2 & 0,0 & 0,0 & 0,0 \\
\hline & Campo & 87 & 72,7 & 22,2 & 0,2 & 0,0 & 0,0 & 0,0 \\
\hline
\end{tabular}

* Média de quatro repetições.

durante o transporte, pois servem de fonte de inóculo para disseminação do fungo a longas distâncias.

Uma das formas de retardar a disseminação de $M$. fijiensis é impedir o transporte de mudas infetadas e o uso das folhas para proteger os frutos de ferimentos durante o transporte e, ao mesmo tempo, utilizar produtos químicos para desinfestação das embalagens e dos veículos nas áreas contaminadas antes de saírem em direção às áreas livres da doença (Stover, 1977).

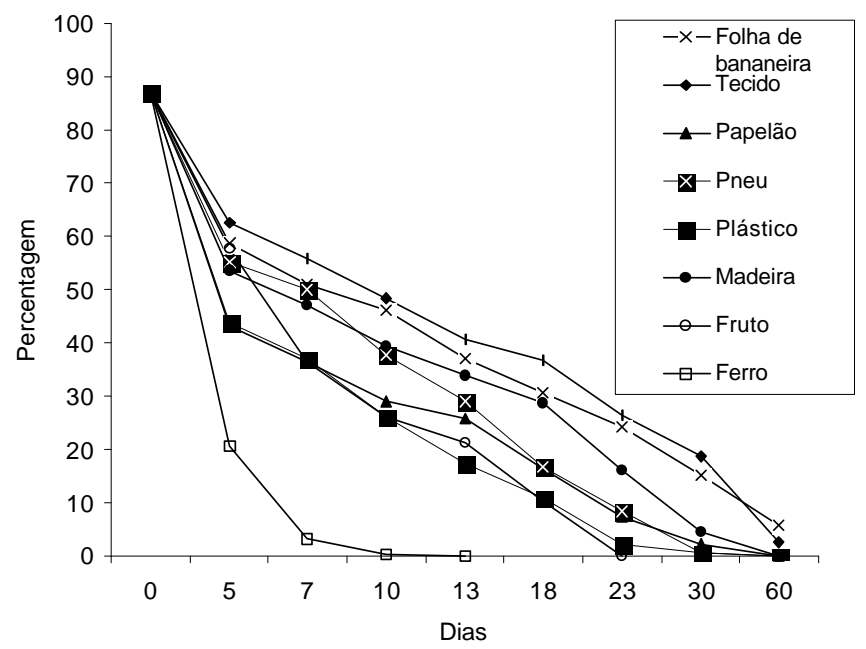

FIG. 1 - Sobrevivência de conídios de Mycosphaerella fijiensis em diferentes materiais.
Calvo \& Romero (1998) encontraram ascósporos de M. fijiensis até uma distância de $50 \mathrm{Km}$, a partir de uma única fonte de inóculo. Enquanto, Stover (1980) observou que os conídios facilmente se desprendem com o vento dos conidióforos e são levados a longas distâncias pela corrente de ar. A facilidade de disseminação dos esporos de $M$. fijiensis associada à longevidade destes, quando aderidos aos diferentes materiais usados na confecção de caixas para transporte de frutas, aos veículos, às roupas dos operários e às folhas e cascas dos frutos da bananeira, implica em dificuldades no estabelecimento de barreiras efetivas que impeçam sua disseminação para o restante do país. Dessa forma, todos os esforços das autoridades na emissão de portarias e no estabelecimento de barreiras fitossanitárias, provavelmente, apenas retardarão a entrada do patógeno em áreas indenes.

\section{REFERÊNCIAS BIBLIOGRÁFICAS}

AMORIM, L. Disseminação. In: Bergamin Filho, A., Kimati, H. \& Amorim, L. (Eds.) Manual de Fitopatologia, 3ae ed., Agronômica Ceres, São Paulo, 1995. pp. 267-293.

CALVO, C. \& ROMERO, R. Evaluación del gradiente de dispersión de la enfemedad de la Sigatoka negra del banano (Musa AAA). Corbana 23:51-56. 1998.

DHINGRA, O.D. \& SINCLAIR, J.B. Basic Plant Pathology Methods. Lewis Publishers, Boca Raton, Florida. 1995.

GASPAROTTO, L., PEREIRA, J.C.R. \& TRINDADE, D.R. Situação atual da Sigatoka negra da bananeira no Brasil. Fitopatologia Brasileira 26:449. 2001. (Resumo).

GONZÁLES, M. Metodología para la manipulación y cultivo in vitro 
de Mycosphaerella fijiensis. Manejo Integrado de Plagas 53:iiv. 1999.

HANADA, R.E., GASPAROTTO, L. \& PEREIRA, J.C.R. Esporulação de Mycosphaerella fijiensis em meio de cultura. Fitopatologia Brasileira 27:170-173. 2002.

JACOME, L.H. \& SCHUH, W. Spore production and artificial inoculation techniques for Mycosphaerella fijiensis var. difformis. Tropical Agriculture 70:33-38. 1993a.

JACOME, L.H. \& SCHUH, W. Effect of temperature on growth and conidial production in vitro, and comparison of infection and agressiveness in vivo among isolates of Mycosphaerella fijiensis var. difformis. Tropical Agriculture 70:51-59. $1993 \mathrm{~b}$.

KRANZ, J., SCHMUTTERER, H. \& KOCH, W. Enfermedades, Plagas y Malezas de los Cultivos Tropicales. Verlag Paul Parey, Berlín y Hamburgo. 1982.
MOSEMAN, J.G. \& POWERS, H.R. Functions and longevity of cleistothecia of Erysiphe graminis f. sp. hordei. Phytophatology 47:33. 1957.

PEREIRA, J.C.R., GASPAROTTO, L., COELHO, A.F.S. \& URBEN A.B. Ocorrência da Sigatoka negra no Brasil. Fitopatologia Brasileira 23:295. 1998. (Resumo).

STOVER, R.H. Sigatoka leaf spots of banana and plantains. Tropical Agriculture Research Services 64:750-756. 1980.

STOVER, R.H. Banana (Musa spp.). In: Hewitt, W.B. \& Chiarappa, L. (Eds.) Plant health and quarantine in international transfer of genetic resources. CRC Press., Cleveland. 1977. pp. 71-79.

SUSSMAN, A.S. \& HALVARSON, H.O. Spores: Their Dormancy and Germination. Harper \& row, New York. 1966.

YOUNG, H.C. \& WADSWORTH, D.F. Collection and race isolation of wheat leaf rust in Oklahoma. Phytopatology 43:33. 1953. 